\title{
The LaLiMo Trial: lamotrigine compared with levetiracetam in the initial 26 weeks of monotherapy for focal and generalised epilepsy-an open-label, prospective, randomised controlled multicenter study
}

\author{
Felix Rosenow, ${ }^{1}$ Carmen Schade-Brittinger, ${ }^{2}$ Nicole Burchardi, ${ }^{2}$ Sebastian Bauer, ${ }^{1}$ \\ Karl Martin Klein, ${ }^{1}$ Yvonne Weber, ${ }^{3,4}$ Holger Lerche, ${ }^{3,4}$ Stefan Evers, ${ }^{5}$ \\ Stjepana Kovac, ${ }^{5}$ Susanne Hallmeyer-Elgner, ${ }^{6}$ Götz Winkler, ${ }^{7}$ Joachim Springub, ${ }^{8}$ \\ Mathias Niedhammer, ${ }^{9}$ Erhard Roth, ${ }^{10}$ Ilonka Eisensehr, ${ }^{11}$ Jörg Berrouschot, ${ }^{12}$ \\ Stephan Arnold, ${ }^{13}$ Michael Schröder, ${ }^{14}$ Anja Beige, ${ }^{14}$ Wolfgang H Oertel, ${ }^{1}$ \\ Adam Strzelczyk, ${ }^{1}$ Anja Haag, ${ }^{1}$ Philipp S Reif, ${ }^{1}$ Hajo M Hamer, ${ }^{1}$ for the LaLiMo Study \\ Group*
}

\begin{abstract}
- An additional material is published online only. To view this file please visit the journal online (http://dx.doi.org/ 10.1136/jnnp-2011-301999).

For numbered affiliations see end of article.
\end{abstract}

\section{Correspondence to}

Professor Felix Rosenow, Epilepsy Center Hessen, Department of Neurology, University Hospitals Giessen \& Marburg, Baldingerstrasse, 35043 Marburg, Germany; rosenow@med.uni-marburg.de

\section{*The LaLiMo Study Group (http://www.lalimo.de) participants are listed in appendix 1. \\ PSR and HMH contributed equally to this work.}

Received 8 December 2011 Revised 22 February 2012 Accepted 27 February 2012

Published Online First 17 May 2012

\begin{abstract}
Background Of the newer antiepileptic drugs, lamotrigine (LTG) and levetiracetam (LEV) are popular first choice drugs for epilepsy. The authors compared these drugs with regard to their efficacy and tolerability in the initial monotherapy for epilepsy.
\end{abstract}

Methods A randomised, open-label, controlled, parallel group, multicenter trial was conducted to test the superiority of the LEV arm over the LTG arm. The primary endpoint was the rate of seizure-free patients in the first 6 weeks (two-sided Fisher's exact test, $\alpha=0.05$, intent-totreat set). Furthermore, efficacy, tolerability and quality of life were evaluated. The authors included 409 patients aged $\geq 12$ years with newly diagnosed focal or generalised epilepsy defined by either two or more unprovoked seizures or one first seizure with high risk for recurrence. Patients were titrated to $2000 \mathrm{mg} /$ day of LEV or $200 \mathrm{mg} /$ day of LTG reached on day 22 or 71 , respectively. Two dose adjustments by 500/50 mg were allowed.

Results The proportions of seizure-free patients were $67.5 \%$ (LEV) versus $64.0 \%$ (LTG) 6 weeks after randomisation ( $p=0.47$ ), and $45.2 \%$ (LEV) versus $47.8 \%$ (LTG) during the whole treatment period of 26 weeks. The HR (LEV vs LTG) for seizure-free time was 0.86 (95\% $\mathrm{Cl}, 0.61$ to 1.22). Adverse events occurred in $74.5 \%$ (LEV) versus $70.6 \%$ ( $L T G)$ of the patients $(p=0.38)$. Adverse events associated with study discontinuation occurred in 17/204 (LEV) versus 8/201 (LTG) patients ( $p=0.07$ ). Conclusions There were no significant differences with regard to efficacy and tolerability of LEV and LTG in newly diagnosed focal and generalised epilepsy despite more rapid titration in the LEV arm.

Clinical trial registration number ClinicalTrials.gov identifier NCT00242606.

\section{INTRODUCTION}

Epilepsy is a chronic neurological disorder with a prevalence of about $0.5 \%$ and incidence rates of 70 per 100000 in children and adolescents, 30 per 100000 in adults aged 20-64 years and 100 per
100000 in older people aged 65 years and older. ${ }^{1}$ In $\geq 20 \%$ of newly diagnosed patients, the epilepsy syndrome is unknown when treatment is initiated..$^{2}$ At the same time, these patients are likely to take the initial monotherapy for a prolonged period of time because it will render about $50 \%$ of them seizure free ${ }^{34}$ while discontinuing the medication will increase the risk of recurrence by about $50 \%$. 5 The choice of the initial antiepileptic drug (AED) is, therefore, of great importance and, ideally, it should have high and broad spectrum efficacy as well as good short- and long-term tolerability and safety, have the ability to be rapidly titrated to effective doses, and have no or little interactions with other drugs or metabolism in general. The Standard and New Antiepileptic Drug-trial (SANAD) investigators concluded that lamotrigine (LTG) is the drug of first choice in focal epilepsies because it showed the highest long-term retention rate. ${ }^{7}$ In generalised or unclassified epilepsy, LTG was less efficacious than valproic acid (VPA) but was equally well tolerated and is still considered the drug of first choice in women of childbearing age, because of its relatively low teratogenicity. ${ }^{8}$

The SANAD trial did not include levetiracetam (LEV) which was first marketed well after study onset. LEV, however, rapidly gained wide acceptance because it has many pharmacokinetic characteristics of an ideal AED. The compound is approved in patients aged 1 month and older, can be rapidly titrated and appears to be void of clinically relevant pharmacokinetic interactions. Serious adverse events (AEs) were rare but LEV does cause relevant $\mathrm{AEs}$ such as tiredness and behavioural and psychiatric disturbances.

Recent comparative trials have shown similar efficacy and tolerability in comparison with extended release carbamazepine $(\mathrm{CBZ})^{9}{ }^{10}$ and VPA. ${ }^{10}$

Immediate seizure control will benefit the patient and society as it will lower epilepsy-related costs, work absenteeism, stigma and reduce interference with quality of life (QoL) as well as the 
time to regain driving permission. ${ }^{11-13}$ Therefore, the possibility of rapid titration is a potential advantage of LEV over LTG. However, the two AEDs have not yet been directly compared in a controlled prospective trial. We, therefore, conducted an active comparator study to evaluate the efficacy and tolerability of LEV and LTG as initial treatment in patients aged 12 years or older with newly diagnosed focal, generalised or unclassified epilepsy.

\section{METHODS}

\section{Patients}

Patients were eligible for the study if they were newly diagnosed with focal, generalised or unclassified epilepsy defined by either two or more unprovoked seizures or one first seizure with high risk for recurrence, such as a lesion on cerebral imaging, a focal neurological deficit, based on ictal symptomatology, or a pathological EEG (either interictal epileptiform discharges or focal slowing). ${ }^{14} 15$ These inclusion criteria followed a recent proposal for a revised definition of epilepsy. ${ }^{16}$ Patients with non-epileptic seizures, auras or absences only, as well as patients with acute symptomatic seizures (occurring within 14 days of an acute brain injury such as a stroke), were excluded. We included patients already receiving one AED at enrolment. Per protocol, this AED was tapered off during the first 3 weeks of the trial by reducing the dose by one-quarter on days 1, 8, 15 and discontinuing it on day 22. A complete list of the eligibility criteria is provided in the online supplementary material.

The study protocol was approved by the relevant ethics committees before patient enrolment commenced. All participants provided written informed consent before entering the study. This trial was registered with http://ClinicalTrials.gov, identifier NCT00242606.

\section{Study design and treatment}

This was a randomised, open-label, active-controlled, parallel group, multicenter monotherapy study. Recruitment was performed by 58 board certified neurologists working either in outpatient departments of hospitals or in private neurological practices in Germany. Patients were randomised in a 1:1 ratio to receive either LEV or LTG, both in normal (not in extendedrelease) formulation, according to a computer-generated balanced randomisation list. Stratification criteria were age (12-15 vs $\geq 16$ years), type of epilepsy (generalised vs focal vs not classified) and centre. Evaluations were performed at visits after 4, 6, 11 and 26 weeks of treatment.

LEV was started with $500 \mathrm{mg} /$ day and the dose was raised by $500 \mathrm{mg} /$ week, while LTG was started with $25 \mathrm{mg}$ and the dose was initially increased by $25 \mathrm{mg} / 2$ weeks and by $50 \mathrm{mg} / 2$ weeks once a daily dose of $100 \mathrm{mg}$ was reached. Target daily doses of $2000 \mathrm{mg}$ of LEV and $200 \mathrm{mg}$ of LTG were to be achieved on day 22 and 71, respectively. In patients under the weight of $50 \mathrm{~kg}$, target daily doses were reduced to $1500 \mathrm{mg}$ of LEV and $150 \mathrm{mg}$ of LTG. After reaching the target dose, two dose adjustments by $500 \mathrm{mg}$ (LEV) or $50 \mathrm{mg}$ (LTG) were allowed depending on seizure control and tolerability. The entire treatment period per patient was 26 weeks. Subsequently patients either remained on LEV or LTG or were switched to another AED at the discretion of their treating neurologists.

\section{Study outcomes}

The primary endpoint was seizure freedom in the first 6 weeks after randomisation, calculated as the rate of seizure-free patients per group in the corresponding period. Secondary endpoints were seizure freedom during the last 16 weeks of the trial and during the complete treatment period calculated as the rate of seizure- free patients between week 11 and the end of treatment, and randomisation and the end of treatment, respectively.

Seizure-free time was calculated from randomisation to the first seizure documented during the treatment period of 26 weeks. Retention time was calculated as the interval from randomisation to the regular end of treatment (26 weeks) or the premature end of treatment.

The number and type of any seizure and AEs were documented using a patient-diary. AEs were documented according to WHO criteria. QoL was assessed using the Quality of Life in Epilepsy 10 (OOLIE-10) questionnaire, which has been shown to discern differences among treatments in clinical trials, ${ }^{17}{ }^{18}$ and changes were calculated from baseline to the end of treatment. Efficacy, tolerability and QoL were evaluated at all visits. Blood samples were collected initially before treatment was started, at 6 weeks and at the end of the study period to calculate creatinine clearance. Subgroup analyses were performed for patient groups frequently considered separately in clinical trials or management: patients aged $12-17$ versus $18-59$ versus $\geq 60$ years, patients with focal versus generalised or unclassified epilepsy, and patients with a single seizure and high risk of seizure recurrences versus those with two or more seizures. These subgroups reportedly have different recurrence rates ${ }^{11} 12$ and were thought to potentially show different responses to the two drugs tested. Data from a subset of 80 patients were acquired for a pharmacoeconomic evaluation, the results of which will be reported separately.

\section{Statistical analysis}

The study was designed to test for superiority of LEV over LTG concerning the primary endpoint of seizure freedom in the first 6 weeks of the trial. We estimated that 183 patients in each treatment group were required to achieve $80 \%$ power $(p=0.05$, two-sided) to detect a clinically relevant difference of $15 \%$ in the seizure-free patients' rate, assuming a rate of $50 \%$ in the LTG arm. ${ }^{19}$ With a dropout rate of $10 \%, 203$ patients were required to be randomised per group.

The analyses of seizure freedom in the first 6 weeks, in the last 16 weeks and during the whole study were performed using Fisher's exact tests, relative risks and risk differences in the intent-to-treat (ITT) set including all patients randomised. Premature discontinuation from the study without documented seizure is a potential bias to the results, as a discontinuation might have been caused by having experienced seizures. Therefore, the following imputation strategy was applied: in the primary analysis, patients who discontinued treatment before week 6 were assumed to have had a seizure irrespective of whether a seizure was documented during the study participation or not. In the analyses of the other seizure-free patients' rates, discontinuation from treatment before week 24 was assessed as due to seizure. Efficacy outcomes were also analysed in the per-protocol (PP) set, as defined in the study protocol, where patients with major protocol violations (patients falling under the minimal weight, pretreatment $>6$ months, treatment duration $<24$ weeks, deviation from target daily dose by more than $1000 \mathrm{mg}$ of LEV or $100 \mathrm{mg}$ of LTG) were not considered.

The log-rank test was performed to compare the Kaplan-Meier estimates of seizure-free time and retention time, and the unadjusted Cox proportional hazards model was used to investigate the impact of study medication.

Differences between treatment groups in QoL were analysed with the Wilcoxon-Mann-Whitney test. Safety analyses were based on the set of patients randomised who took at least one dose of study medication. $\chi^{2}$ Tests were performed to assess 
tolerability and safety (on the basis of the reported AEs), and in the post-hoc subgroup analyses. All reported $p$ values were two sided. Statistical analyses were performed using the SAS statistical package, V.9.1.3. The analyses of secondary endpoints and of the post-hoc subgroups have to be considered as descriptive statistics.

\section{RESULTS}

From March 2005 to October 2007, 409 patients (LEV=206, $\mathrm{LTG}=203$ ) were randomised in the study (figure 1). Baseline demographic and clinical characteristics are given in table 1. All patients randomised were included in the ITT analysis $(n=409)$; 164 patients were considered protocol violators; consequently, 245 patients $(\mathrm{LEV}=116, \mathrm{LTG}=129)$ remained for the PP analysis.

The results of all seizure-free rates are summarised in table 2. The proportions of seizure-free patients at 6 weeks after randomisation in the ITT set were $67.5 \%$ in the LEV arm and $64.0 \%$ in the LTG arm ( $p=0.47)$. The chance for seizure freedom after 6 weeks of treatment was increased by $5 \%(95 \% \mathrm{CI}-8$ to $21)$ in the LEV arm; the absolute difference was 3.4 (95\% CI -5.8 to 12.6). In the PP set, seizure-free rates changed to $83.6 \%$ (LEV) versus $79.8 \%$ (LTG) $(p=0.51)$. The proportions of seizure-free patients during the last 16 weeks of the maintenance period in the ITT set were $51.9 \%$ (LEV) and $55.7 \%$ (LTG) ( $p=0.49$ ), and $45.2 \%(\mathrm{LEV})$ and $47.8 \%$ (LTG) $(\mathrm{p}=0.62)$ during the entire 26 weeks of the study.

The seizure-free time is given in figure 2 . The median time to first seizure could not be calculated because the number of events was insufficient. There was no statistically significant difference between the two treatments ( $\mathrm{HR}=0.86,95 \%$ CI 0.61 to $1.22, \mathrm{p}=0.40)$.

The retention rate did not differ significantly between the LEV and LTG arms (63.1\% vs 71.4\%; p=0.07). Correspondingly, the HR (LEV vs LTG) for the retention time was 1.34 (95\% CI, 0.95 to $1.88, \mathrm{p}=0.10$ ).

Assessment of QoL at the beginning of the treatment (LEV mean: 2.5, SD: 0.9, $\mathrm{n}=148$; LTG mean: 2.5, SD: 0.9, $\mathrm{n}=155$ ) and at the end of treatment (LEV mean: 2.2, SD: 0.9, $\mathrm{n}=148$; LTG mean: 2.2, SD: $0.9, \mathrm{n}=155$ ) was very similar in both treatment groups. Consequently, changes in QoL (beginning to end of treatment) were not significantly different $(p=0.69)$.

Table 3 shows the most common AEs per group. AEs occurred in $74.5 \%$ (LEV) versus $70.6 \%$ (LTG) of the patients ( $p=0.38$ ). Tiredness and aggression occurred significantly more often in the LEV arm than in the LTG arm. Study discontinuation due to AEs occurred not significantly more frequently in the LEV arm (17/ 204 patients) than in the LTG arm (8/201 patients; $p=0.07)$.

A total of 41 serious AEs in 35 patients were reported. Seventeen serious AEs (in 15 patients) occurred in the LTG arm and 24 (in 20 patients) in the LEV arm $(p=0.40)$. Most serious AEs were not suspected to be related to study medications.

\section{Post-hoc subgroup analysis}

Efficacy and tolerability of LEV and LTG were not significantly different in any of the subgroups analysed: patients aged 12-17 versus $18-59$ versus $\geq 60$ years; patients with focal versus
Figure 1 Patient disposition (as recommended by the CONSORT 2010 statement). LEV, levetiracetam; LTG, lamotrigine.

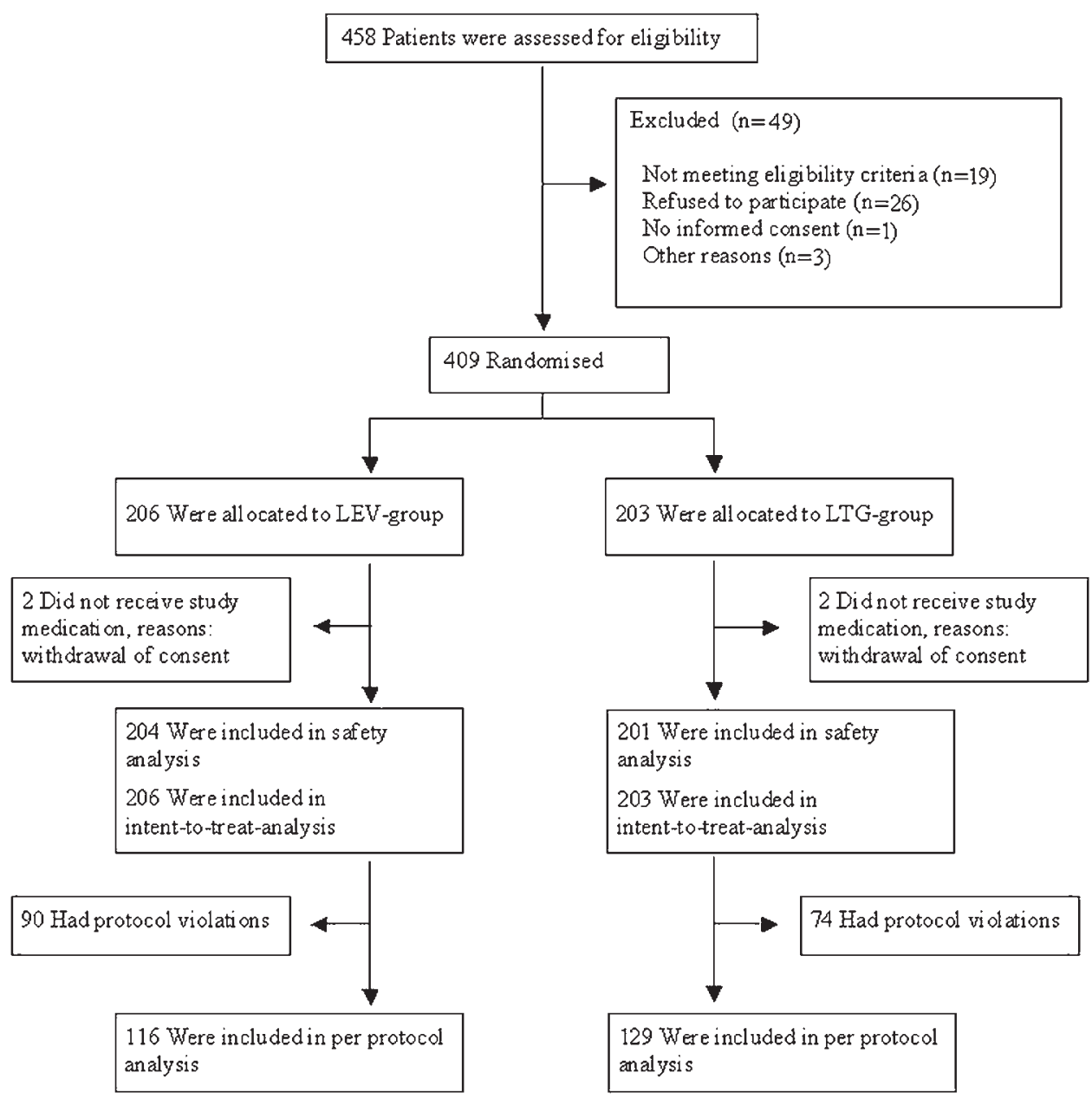


Table 1 Baseline characteristics

\begin{tabular}{llll}
\hline Characteristic & $\begin{array}{l}\text { Levetiracetam } \\
(\mathbf{n}=\mathbf{2 0 6})\end{array}$ & $\begin{array}{l}\text { Lamotrigine } \\
(\mathbf{n}=\mathbf{2 0 3})\end{array}$ & $\begin{array}{l}\text { Total } \\
(\mathbf{n = 4 0 9 )}\end{array}$ \\
\hline Age & & & \\
$\quad$ Median (range) (years) & $39.5(12-84)$ & $37.0(13-80)$ & $38.0(12-84)$ \\
Age groups (years) & $\mathrm{n}(\%)$ & $\mathrm{n}(\%)$ & $\mathrm{n}(\%)$ \\
$\quad 12-15$ & $11(5.3)$ & $9(4.4)$ & $20(4.9)$ \\
$\geq 16$ & $195(94.7)$ & $194(95.6)$ & $389(95.1)$ \\
$12-17$ & $17(8.3)$ & $16(7.9)$ & $33(8.1)$ \\
$18-59$ & $154(74.8)$ & $155(76.4)$ & $309(75.6)$ \\
$\geq 60$ & $35(17.0)$ & $32(15.8)$ & $67(16.4)$ \\
Sex & & & \\
Female & $92(44.7)$ & $102(50.2)$ & $194(47.4)$ \\
$\quad$ Male & $114(55.3)$ & $101(49.8)$ & $215(52.6)$ \\
Weight groups (kg) & & & \\
$\quad \leq 50$ & $10(4.9)$ & $8(3.9)$ & $18(4.4)$ \\
$>50$ & $196(95.1)$ & $195(96.1)$ & $391(95.6)$ \\
Epilepsy syndromes & & & \\
Focal & $104(50.5)$ & $108(53.2)$ & $212(51.8)$ \\
$\quad$ Generalised & $71(34.5)$ & $73(36.0)$ & $144(35.2)$ \\
$\quad$ Not classifiable & $31(15.0)$ & $22(10.8)$ & $53(13.0)$ \\
First seizure versus epilepsy & & & \\
$\quad$ First seizure & $45(21.8)$ & $40(19.7)$ & $85(20.8)$ \\
Epilepsy & $161(78.2)$ & $161(79.3)$ & $322(78.7)$ \\
Missing & $0(0.0)$ & $2(1.0)$ & $2(0.5)$ \\
\hline
\end{tabular}

generalised or unclassified epilepsy; patients with first seizures and a high risk of recurrence as compared with those with two or more seizures.

However, seizure recurrences were less frequent in the older patients $(7 / 67,10.5 \%)$ as compared with the young $(11 / 33$, $33.3 \%)$ and middle aged $(76 / 309,24.6 \%)$ patients $(p=0.02)$. The same was true for the patients with a first seizure and a high risk of recurrence $(11 / 85,12.9 \%)$ as compared with those with epilepsy (83/322, 25.8\%; $\mathrm{p}=0.01)$. On the contrary, the recurrence rates were similar for focal $(54 / 212,25.5 \%)$ versus generalised or unclassified epilepsy (40/197, 20.3\%, $p=0.21)$. The proportion of patients taking an anticonvulsive drug at the time of study enrolment did not significantly differ between the treatment arms (48/409; LEV 20/206, 9.7\%; LTG 28/203, 13.2\%; $\mathrm{p}>0.05)$ nor show a group-dependent influence on the rate of seizure recurrence during the study.

\section{DISCUSSION}

For the initial monotherapy in patients aged 12 years and older with newly diagnosed focal, generalised or unclassified epilepsy, we found no significant differences between LEV and LTG with

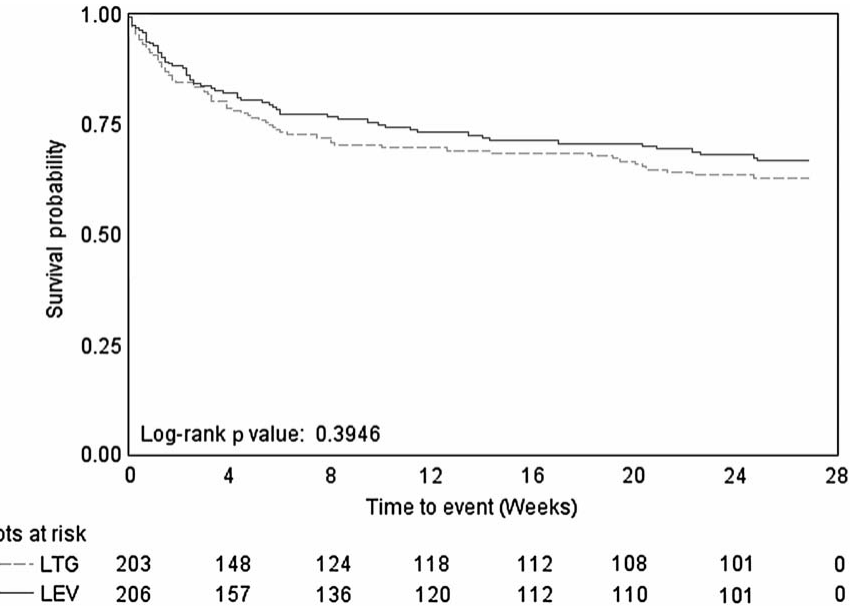

Figure 2 Seizure-free time; levetiracetam (LEV) $n=206$, lamotrigine (LTG) $n=203$.

respect to efficacy, retention and tolerability, although LEV was more rapidly introduced than LTG.

As reported previously, ${ }^{11} 12$ patients aged $\geq 60$ years had a significantly lower rate of seizure recurrence than younger patients, but due to small sample size the validity of this finding is limited. Patients with single seizures in the setting of an increased risk for seizure recurrence according to the proposed new definition of epilepsy ${ }^{16}$ had a significantly lower rate of seizure recurrence than patients who had experienced two or more epileptic seizures at randomisation.

While the seizure-free rate was somewhat higher at 6 weeks after randomisation and while the time to seizure recurrence was longer in the LEV arm, none of these differences reached statistical significance. It could not be shown, to our surprise, that the possibility of a more rapid titration with LEV corresponds to a reduction in seizure recurrence in patients with newly diagnosed epilepsy. This may, in part, be due to the characteristics of our population, which had a much lower rate of seizure recurrence than estimated when calculating the sample size needed, potentially due to the inclusion of $16 \%$ older patients and $21 \%$ of patients after a first seizure. We included patients already receiving one AED at enrolment. Per protocol, this AED was tapered off over the initial 3 weeks of the study and discontinued by day 22. As the proportion of such patients was low in both groups and did not significantly differ between treatment arms nor show a group-dependent influence on the rate of seizure recurrence, it appears highly unlikely that the overall results of the study were affected by the inclusion of such

Table 2 Seizure freedom rates

\begin{tabular}{|c|c|c|c|c|c|}
\hline $\begin{array}{l}\text { ITT } \\
\text { PP }\end{array}$ & $\begin{array}{l}\text { Levetiracetam, N (\%) } \\
\mathbf{n}=\mathbf{2 0 6} \\
\mathbf{n}=\mathbf{1 1 6}\end{array}$ & $\begin{array}{l}\text { Lamotrigine, } N(\%) \\
n=203 \\
n=129\end{array}$ & $\begin{array}{l}\text { Fisher's } \\
\text { exact test }\end{array}$ & RR (95\% Cl) & $\begin{array}{l}\text { Risk difference } \\
(95 \% \mathrm{Cl})\end{array}$ \\
\hline \multicolumn{6}{|l|}{ Primary endpoint } \\
\hline \multicolumn{6}{|l|}{ Seizure freedom after 6 weeks } \\
\hline ITT & $139(67.5)$ & $130(64.0)$ & $p=0.47$ & $1.05(0.92$ to 1.21$)$ & $3.4(-5.8$ to 12.6$)$ \\
\hline PP & $97(83.6)$ & $103(79.8)$ & $p=0.51$ & $1.05(0.93$ to 1.18$)$ & $3.8(-5.9$ to 13.4$)$ \\
\hline \multicolumn{6}{|l|}{ Secondary endpoints } \\
\hline Seizure freedom during the last 16 weeks (ITT) & $107(51.9)$ & $113(55.7)$ & $p=0.49$ & $0.93(0.78$ to 1.12$)$ & $-3.7(-13.4$ to 5.9$)$ \\
\hline Seizure freedom during the last 26 weeks (ITT) & $93(45.2)$ & $97(47.8)$ & $p=0.62$ & $0.94(0.77$ to 1.16$)$ & $-2.6(-12.3$ to 7.0$)$ \\
\hline
\end{tabular}

ITT, intent-to-treat; PP, per-protocol. 
Table 3 Most common adverse events (events counted once per patient)

\begin{tabular}{lllll}
\hline & $\begin{array}{l}\text { LEV }(\mathbf{n}=\mathbf{2 0 4}) \\
\mathbf{n}(\%)\end{array}$ & $\begin{array}{l}\text { LTG }(\mathbf{n}=\mathbf{2 0 1}) \\
\mathbf{n}(\%)\end{array}$ & $\begin{array}{l}\text { Total }(\mathbf{n}=\mathbf{4 0 5}) \\
\mathbf{n}(\%)\end{array}$ & $\begin{array}{l}\text { LEV vs LTG } \\
\mathbf{p} \text { Value* }\end{array}$ \\
\hline Tiredness & $67(32.8)$ & $33(16.4)$ & $100(24.7)$ & $<0.001$ \\
Headache & $47(23.0)$ & $56(27.9)$ & $103(25.4)$ & 0.47 \\
Vertigo & $35(17.2)$ & $39(19.4)$ & $74(18.3)$ & 0.72 \\
URTI & $35(17.2)$ & $27(13.4)$ & $62(15.3)$ & 0.34 \\
Sleep disorder & $22(10.8)$ & $33(16.4)$ & $55(13.6)$ & 0.17 \\
Aggression & $18(8.8)$ & $5(2.5)$ & $23(5.7)$ & $\mathbf{0 . 0 0 6}$ \\
Depression & $10(4.9)$ & $6(3.0)$ & $16(4.0)$ & 0.32 \\
Asthenia & $10(4.9)$ & $8(4.0)$ & $18(4.4)$ & 0.64 \\
Rash & $9(4.4)$ & $19(9.5)$ & $28(6.9)$ & 0.08 \\
Nausea & $9(4.4)$ & $19(9.5)$ & $28(6.9)$ & 0.08 \\
Diarrhoea & $6(2.9)$ & $10(5.0)$ & $16(4.0)$ & 0.45 \\
Tremor & $6(2.9)$ & $15(7.5)$ & $21(5.2)$ & 0.08 \\
\hline p-values below 0.05 in the descriptive statistics are written in bold. \\
* $\chi^{2}$ test. \\
LEV, levetiracetam; LTG, lamotrigine; URTI, upper respiratory tract infection.
\end{tabular}

patients. The proportion of patients with protocol violations was quite high at $40.1 \%(n=165)$. Most of the patients were excluded from the PP set for not achieving the minimum treatment duration of 24 weeks ( $n=134) ; 57$ of them dropped out in the first 6 weeks. This protocol violation had been predefined to generate information about seizure freedom in a patient collective compliant to longer term medication. On comparing proportions of seizure-free patients in the analysis sets, no difference in seizure freedom between patients with short time and longer time medication could be found. Furthermore, the ITT and the PP analyses for the primary endpoint led to identical results regarding risk ratios and risk differences (see table 2). Therefore, it is unlikely, that the protocol violation rate did influence the results of the primary endpoint.

Our subgroup analyses, considering age groups, epilepsy syndrome and the diagnosis of epilepsy versus a first seizure with high recurrence risk, showed no differences regarding the efficacy of the two drugs compared. However, seizure recurrence rates varied among subgroups. It was significantly lower in patients with a first seizure associated with high risk of recurrence as compared with those patients fulfilling the classic definition of epilepsy (occurrence of two or more unprovoked seizures). This finding lends further support to the view to consider these two groups separately, ${ }^{14}$ especially when planning studies of newly diagnosed epilepsy in the future. Furthermore, the likelihood of seizure recurrence was related to age, highest in patients under 18 years and lowest in older patients. Surprisingly, recurrence rate was similar in patients with focal as compared with generalised and unclassified epilepsy syndromes.

Retention was identical in both arms during the first 6 weeks and started to separate in week 7 , being non-significantly lower in the LEV arm later on. Lack of retention was due to lack of tolerability rather than due to lack of efficacy and AEs leading to discontinuation were non-significantly more frequent in the LEV arm. Tiredness and aggression were more frequent in the LEV arm while rash and nausea were twice as frequent in the LTG arm, confirming the side-effect profile of the two drugs revealed in the regulatory trials ${ }^{92-26}$ and clinical practice-data which may help counselling patients regarding the choice of the initial monotherapy. It is possible that side effects would be less prominent if extended release formulations were used.
QoL did not change over the treatment period in both arms. These findings were concordant with results of previously published studies where seizure frequency, but not duration of epilepsy, was associated with a poorer outcome in QoL. ${ }^{27}$ Furthermore, 26 weeks may well be too short to allow for an improvement in GoL because driving restrictions persisted in the majority of the patients and this interval is probably too short to experience reliable seizure control.

The clinical implications of the results of this trial are that a more rapid titration does not necessarily translate into a clinically relevant reduction in the rate of early recurrence, especially when considering initial monotherapy in a population with a relatively low risk of recurrence. This may, however, be different in patients with a high initial seizure frequency. Our study failed to prove superiority of LEV over LTG regarding seizure freedom at 6 weeks after initiation of treatment. Since it was not designed to provide data regarding non-inferiority, this needs to be investigated by further studies.

The decision to include patients with a single seizure and a high risk of seizure recurrence as defined in the proposal of the International League Against Epilepsy and International Bureau for Epilepsy, in $2005{ }^{16}$ is a pragmatic approach and reflects current clinical practice. It has been shown, for instance, that $94 \%$ of patients with a cavernoma and a first seizure will have a recurrence ${ }^{28}$ and, therefore, it would be unjustified to deny anticonvulsive treatment in such circumstances.

The risk of this approach is an overtreatment of patients in cases with benign syndromes and very low recurrence risk, where an anticonvulsive treatment might not be necessary. Therefore, the definition of an 'enduring alteration of the brain that increases the likelihood of future seizures' ${ }^{16}$ used in the new proposal had to be considered carefully. It includes cases with a lesion on brain imaging, a focal neurological deficit, a focal ictal symptomatology or a pathological EEG. ${ }^{14}{ }^{15}$ As epileptiform and non-epileptiform EEG abnormalities have been identified as predictors for seizure recurrence, ${ }^{29} 30$ we included both types of abnormalities in our inclusion criteria. Of interest, using the new definition of epilepsy our study provided evidence that the seizure recurrence rates in patients with a first seizure only is lower, suggesting that in future clinical trials on newly diagnosed epilepsy, patients should be stratified depending on whether or not the new definition of epilepsy is applied.

\section{Author affiliations}

${ }^{1}$ Department of Neurology, Epilepsy Center Hessen, University Hospitals Giessen \& Marburg and Philipps-University Marburg, Germany

${ }^{2}$ Coordinating Center for Clinical Trials (KKS), Philipps-University Marburg, Marburg, Germany

${ }^{3}$ Department of Neurology, University of Ulm, Ulm, Germany

${ }^{4}$ Department of Neurology and Epileptology, University Hospital Tuebingen, Tuebingen, Germany

${ }^{5}$ Department of Neurology, University of Münster, Münster, Germany

${ }^{6}$ Department of Neurology, University of Dresden, Dresden, Germany

${ }^{7}$ Department of Neurology, Leopoldina Hospital, Schweinfurt, Germany

${ }^{8}$ Private Neurological Practice, Westerstede, Germany

${ }^{9}$ Private Neurological Practice, Oldenburg, Germany

${ }^{10}$ Private Neurological Practice, Lich, Germany

${ }^{11}$ Private Neurological Practice, Munich, Germany

${ }^{12}$ Department of Neurology, District Hospital Altenburg, Altenburg, Germany

${ }^{13}$ Department of Neurology, University of Regensburg, Regensburg, Germany

${ }^{14}$ Private Neurological Practice, Munich, Germany

Acknowledgements We would like to thank UCB Pharma for the generous support of our study. We are indebted to the patients for giving their informed consent to participate in the study and for their cooperation throughout the study.

Contributors FR, HMH and CS-B designed the study and wrote the study protocol CS-B, FR and NB drafted and amended the statistical analysis plan and NB was 
responsible for the biometrical analyses in the study. FR, CS-B, HMH and AH reviewed the statistical analysis. FR, SB, KMK, YW, HL, SA, AB, SE, SK, S-HE, GW, JS, MN, ER, $I E, J B, M S, A S$ and PSR had initially approved the protocol and were actively involved in patient recruitment and data collection. SB and PSR were actively involved in data quality assurance and monitoring. FR, NB, CS-B, HMH, WHO, PSR and AS actively contributed to the writing and reviewing of the submitted manuscript. All authors reviewed the manuscript and approved the final version.

Funding UCB Pharma was involved in the study design and provided personnel for regular monitoring visits. UCB Pharma was not involved in data analysis, interpretation of the data and in the decision to submit the paper for publication.

Competing interests This study was sponsored by Philipps-University Marburg, Marburg, Germany. UCB Pharma provided the majority of the funding. Several of the authors have received honoraria and research and travel grants from UCB Pharma, the producer of LEV, and GSK the original producer of LTG.

Ethics approval This study was approved by the ethics committee of the University of Marburg, Germany.

Provenance and peer review Not commissioned; externally peer reviewed.

Data sharing statement For data sharing requests please contact FR (rosenow@ med.uni-marburg.de).

\section{REFERENCES}

1. Forsgren L, Beghi E, Oun A, et al. The epidemiology of epilepsy in Europe a systematic review. Eur J Neurol 2005;12:245-53.

2. King MA, Newton MR, Jackson GD, et al. Epileptology of the first-seizure presentation: a clinical, electroencephalographic, and magnetic resonance imaging study of 300 consecutive patients. Lancet 1998;352:1007-11.

3. Anon. Considerations on designing clinical trials to evaluate the place of new antiepileptic drugs in the treatment of newly diagnosed and chronic patients with epilepsy. Epilepsia 1998;39:799-803.

4. Kwan P, Brodie MJ. Early identification of refractory epilepsy. N Engl J Med 2000;342:314-19

5. Anon. Prognostic index for recurrence of seizures after remission of epilepsy. Medical Research Council Antiepileptic Drug Withdrawal Study Group. BMJ 1993;306:1374-8.

6. Specchio LM, Tramacere L, La Neve A, et al. Discontinuing antiepileptic drugs in patients who are seizure free on monotherapy. $J$ Neurol Neurosurg Psychiatry 2002; $72: 22-5$

7. Marson AG, Al-Kharusi AM, Alwaidh M, et al; SANAD Study group. The SANAD study of effectiveness of carbamazepine, gabapentin, lamotrigine, oxcarbazepine, or topiramate for treatment of partial epilepsy: an unblinded randomised controlled trial. Lancet 2007;369:1000-15.

8. Marson AG, Al-Kharusi AM, Alwaidh M, et al; SANAD Study group. The SANAD study of effectiveness of valproate, lamotrigine, or topiramate for generalised and unclassifiable epilepsy: an unblinded randomised controlled trial. Lancet 2007:369:1016-26.

9. Brodie MJ, Perucca E, Ryvlin P, et al; Levetiracetam Monotherapy Study Group. Comparison of levetiracetam and controlled-release carbamazepine in newly diagnosed epilepsy. Neurology 2007:68:402-8.

10. Pohlmann-Eden B, van Paesschen W, Hallström $Y$, et al. The KOMET study: an open-label, randomized, parallel-group trial comparing the efficacy and safety of levetiracetam with sodium valproate and carbamazepine as monotherapy in subjects with newly diagnosed epilepsy. Epilepsia 2008;49(Suppl 7):448-9.

11. Drazkowski J. An overview of epilepsy and driving. Epilepsia 2007;48(Suppl 9):10-12.

12. Smeets VM, van Lierop BA, Vanhoutvin JP, et al. Epilepsy and employment: literature review. Epilepsy Behav 2007:10:354-62.

13. Strzelczyk A, Reese JP, Dodel R, et al. Cost of epilepsy: a systematic review. Pharmacoeconomics 2008;26:463-76.

14. Haut S, O'Dell C, Shinnar S. Risk factors for developing epilepsy after a first unprovoked seizure. In: Ryvlin P, ed. From First Unprovoked Seizure to Newly Diagnosed Epilepsy. Montrouge, France: John Libbey Eurotext, 2007:37-48.

15. Kim LG, Johnson TL, Marson AG, et al. Prediction of risk of seizure recurrence after a single seizure and early epilepsy: further results from the MESS trial. Lancet Neurol 2006:5:317-22.
16. Fisher RS, van Emde Boas W, Blume W, et al. Epileptic seizures and epilepsy: definitions proposed by the International League Against Epilepsy (ILAE) and the International Bureau for Epilepsy (IBE). Epilepsia 2005;46:470-2.

17. Cramer JA, Arrigo C, Van Hammee G, et al. Comparison between the Q0LIE-31 and derived OOLIE-10 in a clinical trial of levetiracetam. Epilepsy Res 2000;41:29-38.

18. Cramer JA, Perrine K, Devinsky 0 , et al. A brief questionnaire to screen for quality of life in epilepsy: the OOLIE-10. Epilepsia 1996;37:577-82.

19. Brodie MJ, Chadwick DW, Anhut $\mathrm{H}$, et al. Gabapentin versus lamotrigine monotherapy: a double-blind comparison in newly diagnosed epilepsy. Epilepsia 2002; 43:993-1000.

20. Brodie MJ, Overstall PW, Giorgi L. Multicentre, double-blind, randomised comparison between lamotrigine and carbamazepine in elderly patients with newly diagnosed epilepsy. The UK Lamotrigine Elderly Study Group. Epilepsy Res 1999:37:81-7.

21. Brodie MJ, Richens A, Yuen AW. Double-blind comparison of lamotrigine and carbamazepine in newly diagnosed epilepsy. UK Lamotrigine/Carbamazepine Monotherapy Trial Group. Lancet 1995:345:476-9.

22. Chaisewikul R, Privitera MD, Hutton JL, et al. Levetiracetam add-on for drugresistant localization related (partial) epilepsy. Cochrane Database Syst Rev 2001;(1) CD001901.

23. Gamble CL, Williamson PR, Marson AG. Lamotrigine versus carbamazepine monotherapy for epilepsy. Cochrane Database Syst Rev 2006;(1):CD001031.

24. Labiner DM, Ettinger $A B$, Fakhoury $T A$, et al. Effects of lamotrigine compared with levetiracetam on anger, hostility, and total mood in patients with partial epilepsy. Epilepsia 2009;50:434-42.

25. Reunanen M, Dam M, Yuen AW. A randomised open multicentre comparative tria of lamotrigine and carbamazepine as monotherapy in patients with newly diagnosed or recurrent epilepsy. Epilepsy Res 1996:23:149-55.

26. Steiner TJ, Dellaportas Cl, Findley LJ, et al. Lamotrigine monotherapy in newly diagnosed untreated epilepsy: a double-blind comparison with phenytoin. Epilepsia 1999:40:601-7.

27. Guekht AB, Mitrokhina TV, Lebedeva AV, et al. Factors influencing on quality of life in people with epilepsy. Seizure 2007:16:128-33.

28. Josephson CB, Leach JP, Duncan R, et al; Scottish Audit of Intracranial Vascular Malformations (SAIVMs) steering committee and collaborators. Seizure risk from cavernous or arteriovenous malformations: prospective population-based study. Neurology 2011;76:1548-54

29. Shinnar S, Berg AT, Moshe SL, et al. The risk of seizure recurrence after a first unprovoked afebrile seizure in childhood: an extended follow-up. Pediatrics 1996;98:216-25

30. Shinnar S, Kang H, Berg AT, et al. EEG abnormalities in children with a first unprovoked seizure. Epilepsia 1994;35:471-6.

\section{APPENDIX 1}

\section{The LaLiMo Study Group}

Principal investigators (all Germany): S Arnold (München), J Berrouschot (Altenburg), U Bongartz (Köln), J Claßen (Würzburg), S Dorfmüller (Schopfheim), I Eisensehr (München), S Evers (Münster), V Fischer (Neunkirchen), T Freudenberger (Traunstein), CG Haase (Recklinghausen), W Heide (Celle), H Hielscher (Gelsenkirchen), WE Hofmann (Aschaffenburg), U Hoot (Wismar), S Jackowski-Dohrmann (Marburg), P Kalischewski (Leipzig), M Kieslich (Frankfurt), G Kirn (Gladenbach), M Klein (Würzburg), J Klingelhöfer (Chemnitz), I Klyk (Rüsselsheim), A Kowalik (Stuttgart), T Krohn (Wismar), G Kurlemann (Münster), M Lang (Ulm), U Loel (Salzgitter), G Mayer (Schwalmstadt), H Molitor (Würzburg), W Molt (Stuttgart), B Neubauer (Gießen), M Niedhammer (Oldenburg), H Pausch (Marburg), C Pilz (Mannheim), U Reuner (Dresden), N Rigas (Berlin), K Roch (Zwickau), F Rosenow (Marburg), E Roth (Lich), K Rüther (Oberursel), G Schmidt (Ludwigsfelde), M Schröder (Regensburg), A Simonow (Herborn), J Springub (Westerstede), D Städt (Lohr), H Stefan (Erlangen), C Stenzel (Köln-Rodenkirchen), F Stögbauer (Osnabrück), I Sünkeler (Braunfels), U Thumulla (Salzgitter), R Trollmann (Erlangen), S Unglaub (Frankfurt), T Vetter (Schkeuditz), Y Weber (Ulm), B Wild (Nagold), G Winkler (Schweinfurt), A Wirbatz (Gießen), E Wollenhaupt (Dresden), U Zimmerer (Ludwigshafen)

Steering Committee: Bernd A Neubauer (Giessen), Heinz Reichmann (Dresden), Felix Rosenow (Marburg).

Independent Safety Advisory Board (ISAB): Günter Krämer (Zurich), Peter Martus (Berlin), Bernhard J Steinhoff (Kork). 
The LaLiMo Trial: lamotrigine compared with levetiracetam in the initial 26 weeks of monotherapy for focal and generalised epilepsy-an open-label, prospective, randomised controlled multicenter study

Felix Rosenow, Carmen Schade-Brittinger, Nicole Burchardi, Sebastian Bauer, Karl Martin Klein, Yvonne Weber, Holger Lerche, Stefan Evers, Stjepana Kovac, Susanne Hallmeyer-Elgner, Götz Winkler, Joachim Springub, Mathias Niedhammer, Erhard Roth, Ilonka Eisensehr, Jörg Berrouschot, Stephan Arnold, Michael Schröder, Anja Beige, Wolfgang H Oertel, Adam Strzelczyk, Anja Haag, Philipp S Reif, Hajo M Hamer and for the LaLiMo Study Group

J Neurol Neurosurg Psychiatry 2012 83: 1093-1098 originally published online May 17, 2012

doi: 10.1136/jnnp-2011-301999

Updated information and services can be found at:

http://jnnp.bmj.com/content/83/11/1093

\section{These include:}

\section{Supplementary Supplementary material can be found at:}

Material http://jnnp.bmj.com/content/suppl/2012/05/16/jnnp-2011-301999.DC1

References This article cites 27 articles, 5 of which you can access for free at: http://jnnp.bmj.com/content/83/11/1093\#BIBL

Email alerting Receive free email alerts when new articles cite this article. Sign up in the service box at the top right corner of the online article.

Topic
Collections

Articles on similar topics can be found in the following collections

Epilepsy and seizures (846)

Drugs: psychiatry (204)

\section{Notes}

To request permissions go to:

http://group.bmj.com/group/rights-licensing/permissions

To order reprints go to:

http://journals.bmj.com/cgi/reprintform

To subscribe to BMJ go to:

http://group.bmj.com/subscribe/ 